\title{
Termobiología de Bothrops asper (Garman, 1883) en Colombia: ensayos ecofisiológicos
}

\author{
Thermobiology of Bothrops asper (Garman, 1883) in \\ Colombia: ecophysiological tests
}

\author{
Oscar Daniel Medina-Barrios ${ }^{1}$, Éver Edrey Hernández-Cuadrado², \\ Douglas Hernández Vélez ${ }^{3}$
}

\section{Resumen}

Se determinó el rango de temperatura óptimo (RTO) y las temperaturas críticas (CTmax, CTMin) de la tasa respiratoria (TR) y los comportamientos de alimentación (CA) y de defensa (CD) de Bothrops asper en condiciones de cautiverio. Se usaron serpientes adultas $(\mathrm{n}=10)$ con una talla de $137.2 \pm 17.2 \mathrm{~cm}$ y peso de $829.0 \pm 378.9 \mathrm{~g}$, sometidas a temperaturas experimentales entre 10 y $40{ }^{\circ} \mathrm{C}$ y cambios graduales de $3{ }^{\circ} \mathrm{C}$ por minuto, excepto para la captura de alimento $\left(4^{\circ} \mathrm{C}\right.$ por semana). El RTO fue entre 21 y $31^{\circ} \mathrm{C}$ para TR (22.9 $\pm 5.1 \mathrm{resp} / \mathrm{min})$, de 27 a $31{ }^{\circ} \mathrm{C}$ y de 26 a $30{ }^{\circ} \mathrm{C}$ para $\mathrm{CA}$ y $\mathrm{CD}$, respectivamente. Las CTmax y CTMin fueron de $39^{\circ} \mathrm{C}$ y $15.5^{\circ} \mathrm{C}$ para TR, de $34.7^{\circ} \mathrm{C}$ y $20^{\circ} \mathrm{C}$ para CA y de $33^{\circ} \mathrm{C}$ y $21.7^{\circ} \mathrm{C}$ para $\mathrm{CD}$, respectivamente. Las temperaturas y las variables ecofisiológicas estuvieron correlacionadas en la mayoría de los casos $(\mathrm{R}=0.67, \mathrm{p}=0.000)$, observándose mayor sensibilidad a registros térmicos altos. Estos datos demuestran que la temperatura actúa como un modulador de los eventos de la historia de vida y del grado de intensidad y sucesión de los procesos físiológicos y comportamentales de este vipérido. Los resultados también indican que $B$. asper no podría extender su rango de distribución geográfica por encima de los $2000 \mathrm{~m}$ de altitud. Finalmente, se sugiere un grado de amenaza mayor (casi amenazada) para esta especie.

Palabras clave: mapaná; temperatura; respiración; defensa; alimentación; respuesta

${ }^{1}$ Universidad del Tolima, Ibagué, Colombia

${ }^{2}$ Facultad de Medicina Veterinaria y Zootecnia, Universidad del Tolima, Ibagué, Colombia

${ }^{3}$ Programa de Biología, Universidad del Tolima, Ibagué, Colombia

${ }^{4}$ E-mail: ecuadra@ut.edu.co

Recibido: 11 de abril de 2018

Aceptado para publicación: 28 de septiembre de 2018 
The optimal temperature range (RTO) and critical temperatures (CTmax, CTMin) of the respiratory rate (TR), and feeding (CA) and self-defence (CD) behaviour of Bothrops asper in captivity conditions were determined. Adult snakes $(\mathrm{n}=10)$ with a size of $137.2 \pm 17.2 \mathrm{~cm}$ and weight of $829.0 \pm 378.9 \mathrm{~g}$ were used and subjected to experimental temperatures between 10 and $40^{\circ} \mathrm{C}$ and gradual changes of $3{ }^{\circ} \mathrm{C}$ per minute, except for catching their feed $\left(4^{\circ} \mathrm{C}\right.$ per week). The RTO was between 21 and $31^{\circ} \mathrm{C}$ for TR $(22.9 \pm 5.1$ resp/min), 27 to $31^{\circ} \mathrm{C}$ and 26 to $30^{\circ} \mathrm{C}$ for $\mathrm{CA}$ and $\mathrm{CD}$, respectively. CTmax and CTMin were $39^{\circ} \mathrm{C}$ and $15.5^{\circ} \mathrm{C}$ for TR, $34.7^{\circ} \mathrm{C}$ and $20^{\circ} \mathrm{C}$ for $\mathrm{CA}$ and $33^{\circ} \mathrm{C}$ and $21.7^{\circ} \mathrm{C}$ for $\mathrm{CD}$, respectively. Temperatures and ecophysiological variables were correlated in most cases $(\mathrm{R}=0.67, \mathrm{p}=0.000)$, with a higher sensitivity to high thermal recordings. These data showed that temperature acts as a modulator of the events of the life history and the degree of intensity and succession of the physiological and behavioural processes of this viperid. The results also indicate that $B$. asper could not currently extend its geographic distribution range above $2000 \mathrm{~m}$. Finally, a higher threat level (near threatened -NT-) is suggested for this species.

Key words: mapaná; temperature; respiration; defense; feeding; response

\section{INTRODUCCIÓN}

Los factores ambientales son determinantes en el desarrollo y la supervivencia de las especies. Dentro de estos factores es crucial la temperatura, por su incidencia en el movimiento, la alimentación, el cortejo, la respuesta sensorial y la respiración de los vertebrados ectotermos como las serpientes. Entre los aspectos más relevantes de los estudios en biología térmica están los rangos de temperatura óptimos (RTO) y las temperaturas críticas, tanto mínimas como máximas (CTMin, CTMax). Según la IUPS (2003), el RTO es el intervalo de temperaturas donde el animal, o el evento orgánico medido, tiene su máximo desempeño; mientras que las CTMin y CTMax son los registros térmicos más allá de los cuales falla la función fisiológica que se esté estudiando.

De esta forma, el efecto de la temperatura sobre un animal puede ser medido de manera práctica y confiable a través de la evaluación de las respuestas fisiológicas de salida, como el desempeño respiratorio y los comportamientos de defensa y de alimentación. Sin embargo, en la determinación de dicho desempeño se deberán tener presente variables íntimamente ligadas a la ecología térmica de las especies como la historia de vida y su distribución geográfica en relación con la latitud y la altitud (Hernández-Cuadrado, 2009; Ortega y Pérez-Mellado, 2016), buscando una eventual afectación por la interacción de estos aspectos. Bajo esta perspectiva se ha determinado la respuesta fisiológica y comportamental de algunos ectotermos, como reptiles y anfibios tropicales y subtropicales, frente a variaciones térmicas (Huang et al., 2005; Matias y Verrastro, 2018). No obstante, en Colombia son escasos los reportes experimentales de la termobiología de las serpientes, particularmente los del género Bothrops.

Este género comprende alrededor de 45 especies que se distribuyen desde México hasta Argentina (Campell y Lamar, 2004), siendo además el grupo más representativo por accidentes ofídicos en el neotrópico (Otero-Patiño, 2009; Gutiérrez, 2013; INS, 2017). La serpiente B. asper, 
«talla equis», pudridora, mapaná o cuatronarices, es una de las especies más estudiadas del género Brothrops desde el punto de vista epidemiológico (Farsky et al., 2000; Alape-Girón et al., 2008) y de las características químicas y moleculares del veneno (Mack-Wen et al., 2011; MoraObando et al., 2014). En Colombia y en América Latina el accidente ofídico es considerado un evento de salud pública, dada su alta prevalencia (INS, 2014; Yañez-Arenas et al., 2016).

Las dos principales amenazas en Colombia para los vipéridos como las mapanás son la destrucción de su hábitat y la disminución de las poblaciones que conforman sus presas, debido a la actividad antrópica (Lynch, 2012). A pesar de ello, resulta paradójico que se hayan realizado pocos esfuerzos por conocer los aspectos ecológicos, de historia natural o de la biología de estos taxones. El grado de amenaza en la especie no ha sido evaluado (IUCN, 2015), aunque algunos autores lo catalogan como de preocupación menor - LC - (Cisneros-Heredia y Touzet, 2004). Sin embargo, las grandes fluctuaciones térmicas actuales, relacionadas con la afectación de los ecosistemas de sus zonas de distribución, pueden alterar su desempeño $\mathrm{y}$, por tanto, su supervivencia.

Se requiere conocer los diversos aspectos comportamentales y respuestas fisiológicas de salida que pueda presentar $B$. asper en condiciones experimentales, pues las actuales variaciones climáticas están afectando la distribución altitudinal de algunos reptiles. En el presente trabajo se buscó determinar la capacidad de respuesta (desempeño respiratorio, la alimentación y el comportamiento de defensa) ante cambios térmicos bajo condiciones controladas en esta especie. Además, mediante la observación de la respuesta de salida, que evidencia el nivel de afectación fisiológica, se analizó el grado de amenaza de esta serpiente en Colombia.

\section{Materiales y Métodos}

Esta investigación se realizó en las antiguas instalaciones del Serpentario Nacional, ubicado en el municipio de Armero Guayabal (Tolima, Colombia), a una altura de $285 \mathrm{~m}$, temperatura promedio de $28{ }^{\circ} \mathrm{C}$ y humedad relativa (HR) aproximada de $75 \%$. El fotoperiodo fue 12:12, predominante en el lugar de estudio, permitiéndose el flujo de aire en todo momento, lo cual aseguró los niveles adecuados de oxigenación y humedad relativa del sistema. El control de estas variables ambientales y de manejo garantizaban el bienestar de los animales antes, durante y después de los ensayos específicos.

Se utilizaron serpientes adultas $(\mathrm{n}=10)$ de la especie $B$. asper, con una talla de 137.2 $\pm 17.2 \mathrm{~cm}$ (mínimo 102, máximo $165 \mathrm{~cm}$ ), peso de $829.0 \pm 378.9 \mathrm{~g}$ (mínimo 312.5 , máximo $1540 \mathrm{~g})$ y promedio etario de 6.5 años (mínimo 3.5, máximo 8 años). Este último dato fue reportado por el serpentario. Los ejemplares fueron capturados en el medio natural regional y llevadas a confinamiento durante aproximadamente tres meses, previo al inicio de los ensayos de termobiología de este estudio, los cuales se hicieron en el mismo horario (entre las 14:00 a 18:00 horas) durante las 24 semanas de la investigación. En dicho periodo se observaron las indicaciones del protocolo de uso y el manejo de animales experimentales del serpentario.

El experimento se dividió en dos fases: en la primera se determinaron las temperaturas críticas mínimas de la respiración y de los comportamientos de defensa y de alimentación. En la segunda fase se midieron las temperaturas críticas máximas de estos desempeños. En todos los casos se usaron termohigrómetros (KTJ, ref. TA-218D) en el interior del cuarto experimental para controlar y mantener la temperatura (según el caso) y la humedad relativa $(77 \pm 2 \%)$. Los rangos de estos dispositivos eran de 0 a $50{ }^{\circ} \mathrm{C}$ $\left( \pm 0.1^{\circ} \mathrm{C}\right)$ y de 10 a $99 \%( \pm 1 \% \mathrm{HR})$. 


\section{Rango de Temperatura Óptimo}

Se establecieron los rangos de temperatura óptimos (RTO) de la tasa respiratoria - TR - (respiraciones/minuto o movimientos de inflamiento/minuto), del comportamiento de alimentación (CA) y de defensa (CD). El RTO del desempeño respiratorio fue delimitado como el rango de mediciones en el cual no hubo diferencias estadísticamente significativas, tanto para las temperaturas altas como para las bajas, por medio de una prueba $t$. Las observaciones de la TR fueron realizadas a una distancia aproximada de un metro desde la parte superior del contenedor (cubierta transparente) donde se ubicaron los animales para esta prueba. Para los comportamientos de defensa y de alimentación, se tomó como límites críticos el $80 \%$ de desempeño respecto de la temperatura óptima. Esta metodología ha sido previamente usada en el estudio de ectotermos (Huey y Stevenson, 1979; Wilson, 2001; Angilleta Jr et al., 2001; Hernández-Cuadrado, 2009), en los cuales los animales respondieron positivamente a las pruebas respectivas. También se halló el rango de temperatura subóptimo (RTSO), para cada variable en estudio, determinado como el intervalo térmico en el cual la mitad de los animales tuvieron el 50\% de desempeño de dicha variable.

\section{Temperaturas Críticas Mínimas del Des- empeño Respiratorio}

Al inicio de las pruebas se tomó el registro de la tasa respiratoria (respiraciones/ minuto) de las serpientes a la temperatura media de su hábitat $\left(28^{\circ} \mathrm{C}\right)$. Luego, se les ubicó individualmente en un refrigerador industrial (ICASA ILH 950) de $1 \mathrm{~m}^{3}$ aproximadamente, con láminas termoaislantes, para obtener una tasa de descenso térmico de $1{ }^{\circ} \mathrm{C}$ cada $3 \mathrm{~min}$. La parte frontal del refrigerador fue cubierta con una lámina de vidrio de 4 mm para poder observar los movimientos respiratorios. Se controló tanto la temperatura como la humedad relativa en el interior del refrigerador. La CTMin se tomó como el promedio de los registros térmicos en los cuales los ejemplares disminuyeron su tasa respiratoria hasta esta fue imperceptible.

\section{Temperaturas Críticas Mínimas del Comportamiento de Defensa}

Para esta prueba se dejó un lapso de una semana de reposo o recuperación de los animales, teniendo en cuenta que el efecto de la temperatura sobre los ectotermos es instantáneo (Bartholomew, 1979; HernándezCuadrado, 2009; Hernández-Cuadrado et al., 2014). La puerta del refrigerador industrial de experimentación se abría levemente con una vara de madera de $1.5 \mathrm{~m}$ en cuyo extremo se fijó un guante de látex con agua entre 37 y $38^{\circ} \mathrm{C}$, para simular la temperatura corporal promedio de animales endotermos. Esto permitió inducir una respuesta, positiva o negativa, de defensa (ataque), según lo descrito por Lynch (2012). Después de cada respuesta positiva se aplicó el descenso de $1{ }^{\circ} \mathrm{C}$ cada 3 min, tiempo después del cual se estimulaba sucesivamente a la serpiente, hasta alcanzar la CTMin; es decir, el promedio de los registros térmicos en los cuales los ejemplares no mostraron una respuesta positiva.

\section{Temperaturas Críticas Mínimas del Comportamiento de Alimentación}

Este experimento se realizó una semana después de la toma de las CTmin del comportamiento de defensa. En esta prueba se ofreció alimento (un ratón de laboratorio adulto) una vez por semana, disminuyendo la temperatura en $4^{\circ} \mathrm{C}$ por semana. Los ratones tenían un peso entre 15 y $25 \mathrm{~g}$, los cuales eran ofrecidos como presas a las serpientes en proporción a su tamaño corporal. La CTMin se tomó como el promedio de los registros térmicos en los cuales los ejemplares no reaccionaron ante el alimento. 


\section{Temperaturas Críticas Máximas del Des- empeño Respiratorio y Comportamien- tos de Defensa y Alimentación}

Los experimentos para temperaturas altas se realizaron de manera similar a la toma de las CTMin, con la misma duración, pero modificando el cubículo de experimentación para los incrementos de temperatura. Para tal efecto, se construyó una caja de madera tipo tríplex de $1 \mathrm{~m}^{3}$, cuyo interior fue revestido con láminas termoaislantes para estabilizar las temperaturas requeridas, con una tasa de incremento de $1{ }^{\circ} \mathrm{C}$ cada $3 \mathrm{~min}$ (respiración y defensa), tomando como registro térmico inicial la temperatura ambiente $\left(28^{\circ} \mathrm{C}\right)$. Tanto a la caja de tríplex como a su revestimiento se le hicieron pequeños orificios ( 2 $\mathrm{mm}$ ) en la superficie de tres de sus paredes verticales para disipar el exceso de humedad generada por el aumento térmico ( $\mathrm{HR}<80 \%$ ). Para el caso de la prueba de alimentación se hizo un incremento de $4^{\circ} \mathrm{C}$ por semana.

La tasa de aumento térmico se alcanzó calentando agua en un recipiente sobre una estufa eléctrica, la cual fue ubicada debajo del cubículo de tríplex. La cara frontal del cuarto experimental se cubrió con un plástico transparente (de tres milímetros de grosor), resistente a elevadas temperaturas para facilitar la observación de los ejemplares y de las variables estudiadas.

Las CTMax se tomaron como los promedios térmicos en los cuales los animales mostraban taquipnea y se movían rápidamente buscando una salida (tasa respiratoria) o cuando no exhibían las variables estudiadas (comportamientos de defensa y de alimentación). Las temperaturas críticas mínimas y máximas fueron relacionadas con patrones de distribución geográfica (Luttershmidt y Hutchison, 1997; Hernández-Cuadrado, 2013).

\section{Análisis de Datos}

Se realizó un análisis descriptivo de cada variable y comparaciones entre las distintas temperaturas con el desempeño respiratorio, utilizando la prueba $t$. Se usó la prueba de correlación de Spearman para determinar el grado de asociación entre las temperaturas de los experimentos con las variables fisiológicas en estudio. A los comportamientos de defensa y alimentación (variables cualitativas) se les asignaron valores numéricos (cuantitativos) según los incrementos o descensos de temperatura. Para ello se tuvo en cuenta el efecto sobre el parámetro fisiológico medido, así: $28^{\circ} \mathrm{C}=1$ y a partir de 30 hasta $36{ }^{\circ} \mathrm{C}$ del 2 al 8 . En forma similar, $25^{\circ} \mathrm{C}=2$ y de 24 a $15{ }^{\circ} \mathrm{C}$ de 3 al 12 . A la ausencia del comportamiento respectivo se dio un valor de cero (0). Las pruebas se realizaron con una significancia del $95 \%$ y los resultados se mostraron como la desviación estándar de la media $( \pm \mathrm{DE})$, usando el paquete Statistica v. 7.1.

\section{Resultados}

\section{Rango de Temperatura Óptimo y Tem- peraturas Críticas de la Tasa Respirato- ria}

El RTO para la TR de la especie estuvo entre 21 y $31{ }^{\circ} \mathrm{C}$, sin diferencias significativas entre ellas (Figura 1). La TR tuvo una media de $22.9 \pm 5.1$ respiraciones/minuto (mínima de $16.8 \pm 8.7$ y máxima de $33.4 \pm$ 19.3) (Figuras 2 y 3). Los rangos de temperaturas subóptimas menor y mayor estuvieron entre 20 y $21^{\circ} \mathrm{C}$ y entre 31 y $32^{\circ} \mathrm{C}$, respectivamente. Se halló una correlación positiva fuerte entre las temperaturas experimentales, tanto altas $(\mathrm{R}=0.94, \mathrm{p}=0.000)$ como bajas $(\mathrm{R}=0.981, \mathrm{p}=0.000)$ con la frecuencia respiratoria. La CTMax para el desempeño respiratorio se presentó en promedio a $39^{\circ} \mathrm{C}$, pues a temperaturas superiores las serpientes buscaban una ruta de escape al entrar en estrés térmico. La CTMin para esta misma variable fue de $15.5^{\circ} \mathrm{C}$. 


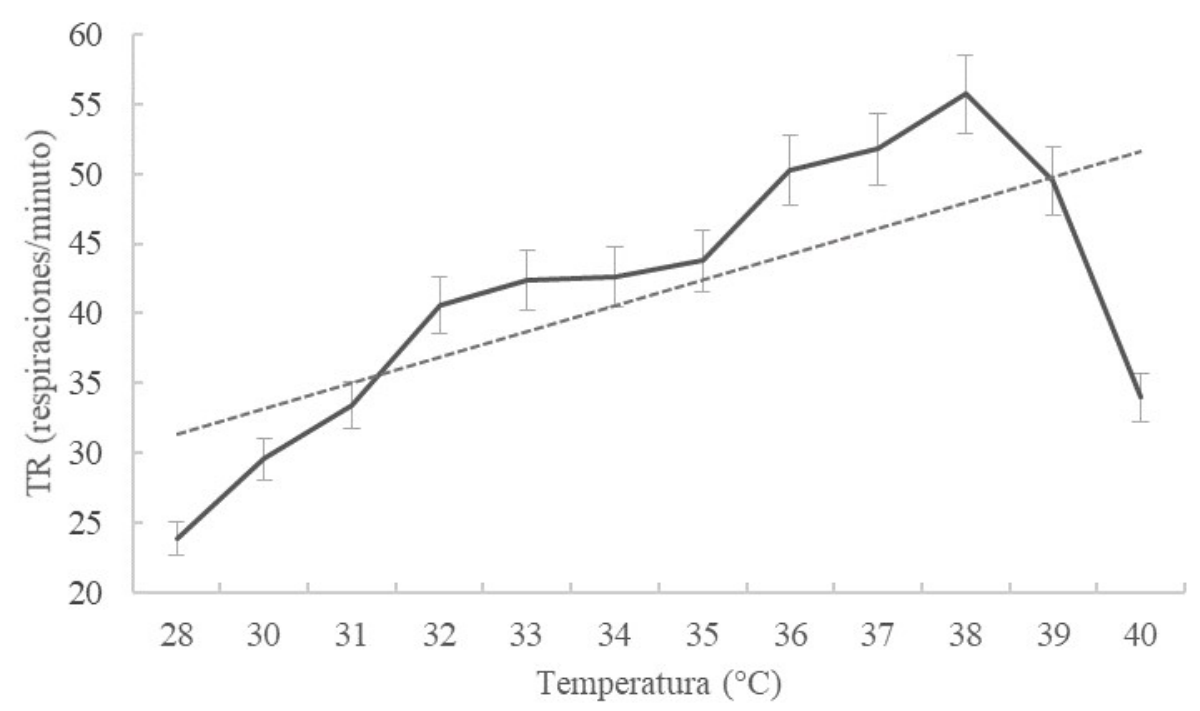

Figura 1. Tasa respiratoria de Bothrops asper en el intervalo de temperatura entre 28 y $40{ }^{\circ} \mathrm{C}$. El trazo punteado corresponde a la línea de tendencia del desempeño

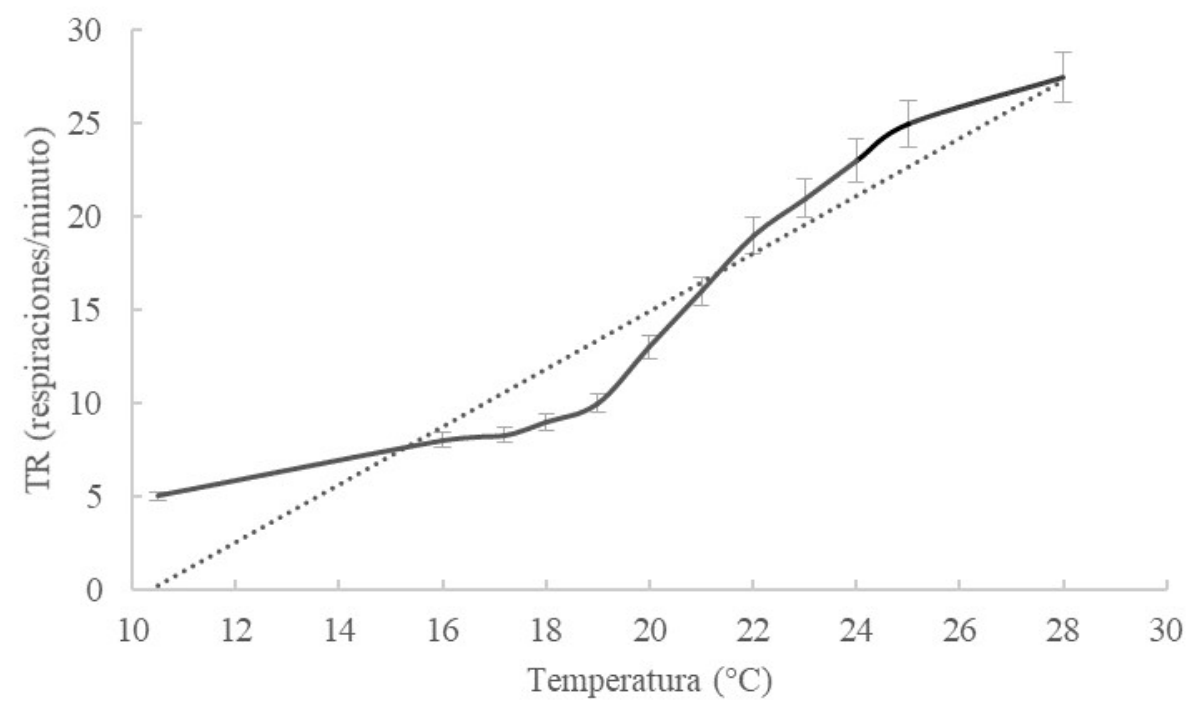

Figura 2. Tasa respiratoria de Bothrops asper en el intervalo de temperatura entre 11 y $28^{\circ} \mathrm{C}$. El trazo punteado corresponde a la línea de tendencia del desempeño 


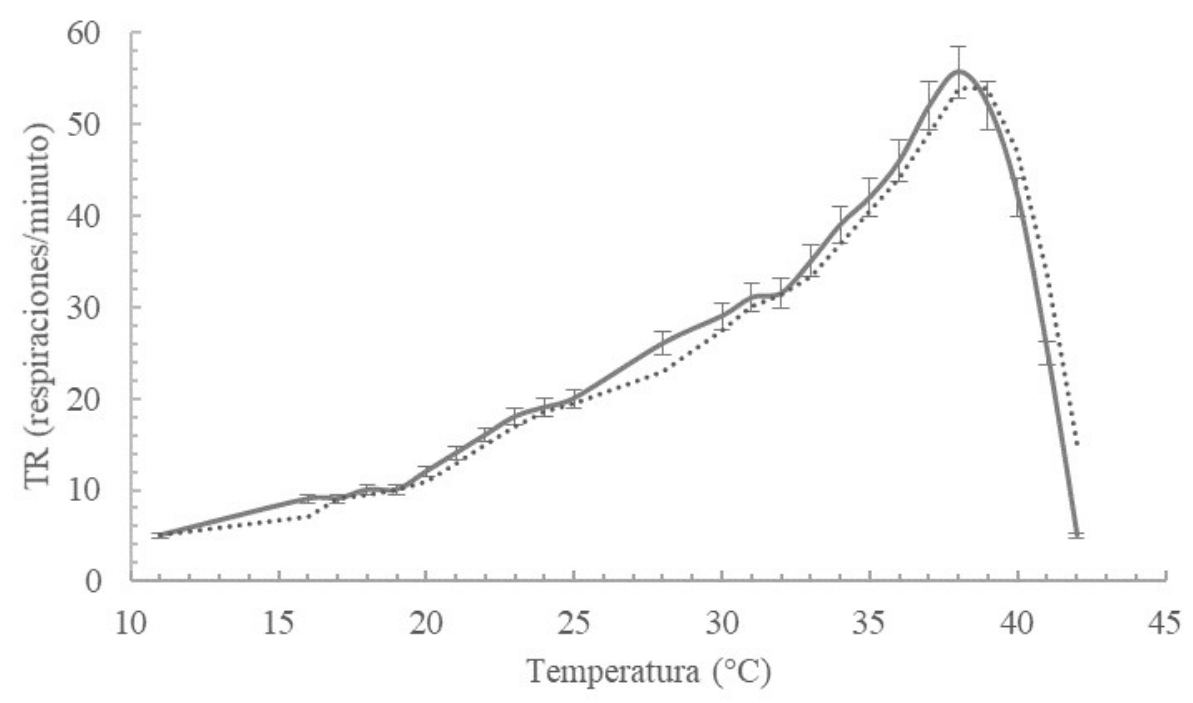

Figura 3. Comportamiento de la tasa respiratoria (respiraciones/minuto) de Bothrops asper en un amplio rango térmico. El trazo punteado corresponde a la línea de tendencia del desempeño

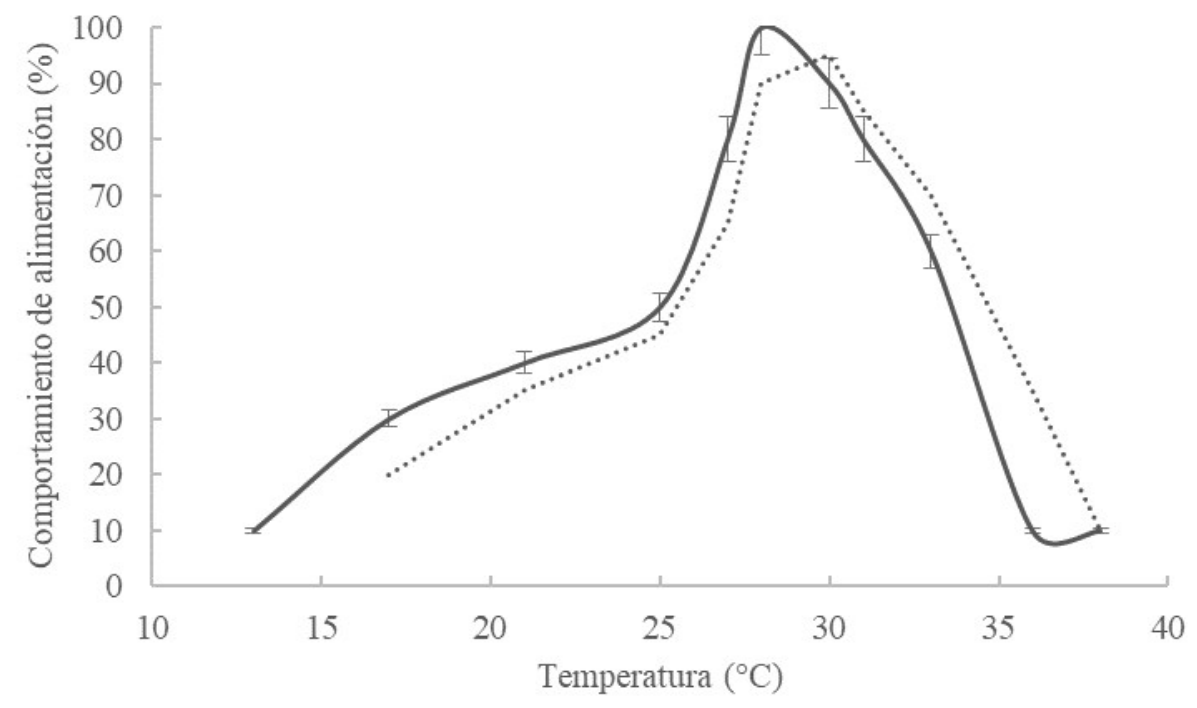

Figura 4. Efecto de la temperatura sobre la captura del alimento en Bothrops asper. El trazo punteado corresponde a la línea de tendencia del desempeño 


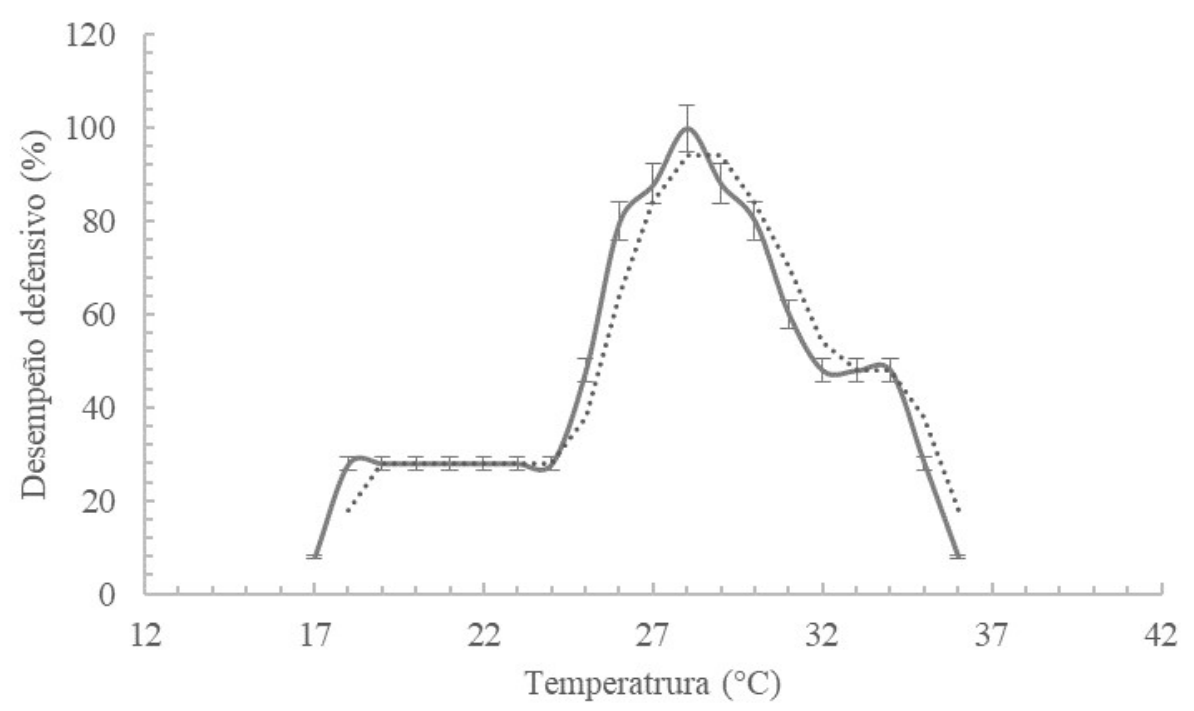

Figura 5. Comportamiento de defensa de Bothrops asper a diferentes temperaturas experimentales. El trazo punteado corresponde a la línea de tendencia del desempeño

\section{Rango de Temperatura Óptimo y Tem- peraturas Críticas del Comportamiento de Alimentación}

El RTO fue entre 27 y $31^{\circ} \mathrm{C}$ (Figura 4). Se obtuvieron dos rangos de temperaturas subóptimas, uno entre 25 y $26.5^{\circ} \mathrm{C}$ y otro entre 30.5 y $33^{\circ} \mathrm{C}$. No hubo correlación significativa entre las temperaturas altas $(\mathrm{R}=-$ $0.500, \mathrm{p}=0.391)$ o bajas $(\mathrm{R}=0.153, \mathrm{p}=0.804)$ con el comportamiento de alimentación (Figura 2). La CTMax para el comportamiento de alimentación se presentó en promedio a $34.7^{\circ} \mathrm{C}$ y la CTMin fue de $20^{\circ} \mathrm{C}$.

\section{Rango de Temperatura Óptimo y Tem- peraturas Críticas del Comportamiento de Defensa}

El RTO estuvo entre 26 y $30^{\circ} \mathrm{C}$ (Figura 5), mostrando un margen moderado de sensibilidad térmica. El $80 \%$ o más de registros positivos estuvo dentro de este rango de temperatura. Los rangos subóptimos de temperatura fueron determinados entre 24 y $26^{\circ} \mathrm{C}$ y entre 30 y $31^{\circ} \mathrm{C}$, en los cuales aproximadamente la mitad de los animales respondieron adecuadamente al experimento. En general, las serpientes exhibieron un comportamiento de evitación en temperaturas subóptimas. No hubo correlación entre las temperaturas experimentales altas y el comportamiento defensivo ( $R=0.67, p=0.071)$; sin embargo, se observó la influencia de la temperatura elevada en la disminución de este comportamiento.

En las temperaturas experimentales bajas se halló una fuerte correlación $(\mathrm{R}=-$ 0.754, $\mathrm{p}=0.004)$. La CTMax registrada fue en promedio de $33^{\circ} \mathrm{C}$ (Figura 4). A partir de los $37^{\circ} \mathrm{C}$, las serpientes buscaban una ruta para escapar de este ambiente. Se observó que el comportamiento de defensa es más acentuado a temperaturas bajas, contrario a lo que se esperaría en un animal cuyo hábitat típico es de tierras medias y bajas. La CTMin registrada para el comportamiento defensivo se dio en promedio a $21.7^{\circ} \mathrm{C}$. 
Discusión

\section{Fisiología Térmica}

La temperatura juega un papel clave en la respiración, captura de presas y respuesta de defensa de $B$. asper. Esta especie presenta un mayor rango de tolerancia a temperaturas menores que la ambiental de su hábitat en Colombia. Posiblemente estas serpientes han seleccionado microhábitats donde la temperatura corporal tiene variaciones reducidas frente a las fluctuaciones del ambiente, como sucede con otros reptiles (Bustos et al., 2013), lo cual es un indicio del grado de adaptación de una especie. Esto permite el desarrollo de mecanismos eficientes del control del enfriamiento, disminuyendo fisiológicamente el ritmo cardiaco, realizando vasoconstricción periférica y adoptando posiciones tales como el enrollamiento (Glanville y Seebacher, 2006). Ello es evidente en la especie estudiada, pues prefiere ambientes muy húmedos o acuáticos, flancos de quebradas o arroyos, reduciendo así el estrés causado por elevadas temperaturas de las áreas donde se distribuye (Wasko y Sasa, 2009). Esto es cierto si se tiene en cuenta que, ante las presiones ambientales de su área de distribución, $B$. asper busca mejorar el balance de agua a través de ajustes fisiológicos, fenotípicos y comportamentales (Saldarriaga-Córdoba et al., 2009; Díaz Ricaurte et al., 2018), lo cual se observa en la mayoría de los reptiles.

Los resultados de esta investigación concuerdan con los planteamientos de Bozinovic (2003), quien indica que la tolerancia a un sobrecalentamiento (hipertermia) es menor que a un shock de baja temperatura (hipotermia). Es así que pueden darse efectos nocivos en los ectotermos por exposición a altas temperaturas que afectarían marcada-mente su comportamiento de defensa y, por tanto, su capacidad de sobrevivencia. Se corrobora que a menor temperatura se da una mayor eficacia en el desarrollo del comportamiento de defensa en $B$. asper. Esto se puede explicar por el hecho de que la temperatura corporal habitual no optimiza simultáneamente los componentes organísmicos en su totalidad, por lo que los sistemas fisiológicos presentarían un orden de importancia en el animal (Huey y Stevenson, 1979). Es decir, en esta serpiente la defensa sería más apremiante que alimentarse, bajo el régimen de temperatura experimental usado. Por tanto, podría decirse que fisiológicamente la especie está adaptada para responder mejor en horas crepusculares y nocturnas, dado que durante las horas de mayor incidencia solar este vipérido se mantiene oculto.

Las temperaturas altas pueden afectar la capacidad de defenderse y alimentarse en $B$. asper, pues parece ser que ocurre una pérdida de la percepción de la profundidad en los movimientos. Ello indica un impacto sobre el sistema nervioso central (SNC), particularmente en las terminaciones que inervan la visión y especialmente el órgano termorreceptor (Seebacher y Franklin, 2005). El número y la velocidad de los ataques (golpes) disminuyeron en el presente estudio con el incremento de la temperatura ambiental, indicando que a nivel muscular también hay una importante afectación. Prueba de esto es que luego de los movimientos defensivos, los animales quedaban completamente inmóviles. En futuros estudios sería recomendable abordar la funcionalidad de las proteínas contráctiles, el intercambio iónico y las proteínas chaperonas $(h s p)$ para corroborar estas observaciones.

Como otros grupos de vertebrados, estas serpientes priorizan la supervivencia antes que cualquier otra actividad. Para temperaturas altas buscan salidas de escape, dejando a un lado otros comportamientos como el alimenticio (Huey y Stevenson, 1979). Estas respuestas podrían estar vinculadas a procesos de dispersión y colonización de áreas con condiciones más favorables, tanto en sentido horizontal como vertical, como ha sucedido con otros grupos de ectotermos (Bustamante et al., 2005; Hernández-Cuadrado et al., 2014). Se ha documentado que 
estos cambios fisiológicos podrían afectar la composición y el efecto biológico del veneno de estas serpientes en áreas geográficas diferentes a las de su distribución actual (AlapeGirón et al., 2008; Saldarriaga-Córdoba et al., 2017), aunque también existen datos que indican lo contrario (Vélez et al., 2017). Ello amerita estudios puntuales.

Con los datos mostrados en la Figura 3 se ratifica que el efecto de las temperaturas altas en ectotermos es más devastador que los causados por temperaturas bajas. Esto puede verse en la pendiente suave de la curva de desempeño respiratorio para las bajas temperaturas y la caída fuerte en esta variable por encima de los $39^{\circ} \mathrm{C}$. A temperaturas bajas las serpientes tuvieron un mejor desempeño respiratorio, pudiendo tolerar hasta $11^{\circ} \mathrm{C}$.

\section{Rango de Temperatura Óptimo (RTO)}

Es probable que el RTO del comportamiento alimenticio de $B$. asper sea más amplio, respecto del defensivo, para tener más posibilidades de alimentarse o de encontrar presas potenciales. A su vez, el estrecho rango para el comportamiento defensivo sería explicado a la luz de sus hábitos sedentarios. Esta característica de permanecer en reposo, o al acecho para no alterar sus funciones fisiológicas y responder de la mejor manera ante posibles presas o predadores, favorece su posibilidad de sobrevivir. La mayor amplitud del RTO del desempeño respiratorio se explicaría por los posibles ajustes metabólicos realizados, buscando extender el rango de tolerancia térmica, como sucede en otros organismos (Leroi et al., 1994). Si logra hacer dichos ajustes, podrá encontrar nuevas temperaturas que le permitan termorregular sin restricciones (Bozinovic, 2003) para responder ante eventuales presas o predadores.

Por otro lado, las coloraciones oscuras de estas serpientes podrían estar asociadas a su hábitat y a su historia natural (HernándezCuadrado, 2009). Es decir, que al preferir microhábitats con temperaturas más bajas que las típicas del ambiente circundante, sus cuerpos deberán tomar colores que permitan una mayor absorción de la radiación del medio. Así, Bozinovic (2003) indica que existe una asociación entre absorción de radiación solar y la coloración de los animales. De este modo se explicaría la mayor ocurrencia de reptiles con coloraciones oscuras en hábitats de bajas temperaturas. En el caso de temperaturas subóptimas donde los animales todavía pueden responder o sobrevivir con cierto grado de estrés, es posible que las serpientes puedan ajustar algunos sistemas fisiológicos y minimizar las pérdidas metabólicas y el riesgo de predación (Huey y Stevenson, 1979).

Los datos obtenidos muestran que para determinar el RTO en B. asper con mayor exactitud no es suficiente considerar el $80 \%$ del desempeño de los parámetros fisiológicos a estudiar. Se sugiere extenderlo a las temperaturas subóptimas para evitar confusiones al momento de analizar las respuestas ante cambios térmicos. También se propone el uso de nuevas metodologías para la determinación de los RTO, incluyendo la prueba del fenómeno de aclimatación térmica. De esta forma, cuando se obtienen las CTMin y CTMax, se toman los dos mejores desempeños en las respectivas temperaturas, tanto superiores como inferiores, teniendo en cuenta que la función fisiológica aún no ha fallado en ese punto, y se hallan las medias de estos desempeños, las cuales podrían ser consideradas como límites para establecer el RTO en forma más objetiva.

\section{Temperaturas Críticas}

Los resultados indican que $B$. asper no soporta una variación térmica muy alta, lo cual explica que esta especie no habita en zonas desérticas o en altitudes moderadas, ya que las condiciones climáticas de estas áreas no llenan los requerimientos térmicos y fisiológicos de esta serpiente (Luttershmidt y Hutchison, 1997; Lynch, 2012). Las temperaturas elevadas pueden causar daños irreversibles a las membranas y proteínas (Sherwood et al., 2012) y pueden tornar a 
estas serpientes más susceptibles a parásitos y a predadores, como ha sido reportado en Clelia clelia y otros ectotermos (Sasa et al., 2009; Bravo y Porzencanki, 2018). Los resultados indican que $B$. asper tiende a buscar una salida de escape y deja de capturar sus presas entre 35 y $39^{\circ} \mathrm{C}$.

A $40{ }^{\circ} \mathrm{C}$ los ejemplares de $B$. asper estudiados mueren o entran en una condición de letargo. Esto fue descrito previamente por Bartholomew (1979) quien indicó que el límite superior del rango de temperaturas que pueden ser toleradas por los animales está cerca a la temperatura en la cual las proteínas llegan a desnaturalizarse. Por tanto, temperaturas a partir de $\operatorname{los} 39^{\circ} \mathrm{C}$ serian letales para estas serpientes.

En temperaturas bajas se observan marcadas diferencias respecto de las exposiciones a temperaturas elevadas. Este estudio corroboró los datos de Cisneros-Heredia y Touzet (2004)' quienes reportan a $B$. asper en Ecuador entre el nivel del mar y los 1720 $\mathrm{m}$ de altitud. De acuerdo con las temperaturas usadas y toleradas en la presente investigación, es probable que individuos de esta especie puedan ampliar su rango de distribución altitudinal hasta los 2000 m; es decir, por encima de lo indicado por Díaz-Ricaurte et al. (2018). No obstante, la capacidad de colonizar estas altitudes estará determinada por la velocidad a la que ocurran los cambios térmicos y otros factores implicados como la fragmentación y degradación de las áreas que habita. Actualmente es poco probable que esta especie pueda llegar a estas altitudes, donde la temperatura promedio es de $12^{\circ} \mathrm{C}$, muy cercana a los $11^{\circ} \mathrm{C}$ de la CTMin de su tasa respiratoria. De ahí que se requiera evaluar el efecto de la temperatura en conjunto con otras variables ambientales para contrastar los datos aquí mostrados.

Los ensayos experimentales del presente estudio indican que los cambios térmicos ambientales pueden afectar la capacidad de $B$. asper para alimentarse, huir y atacar. Esto es evidente en el desempeño respiratorio, donde temperaturas mayores a $39^{\circ} \mathrm{C}$ y menores de $15^{\circ} \mathrm{C}$ les causaron fuertes alteraciones. Así mismo, para el comportamiento defensivo, temperaturas mayores a $33{ }^{\circ} \mathrm{C} \mathrm{y}$ menores de $21^{\circ} \mathrm{C}$ conllevan a una pérdida clara del interés por defenderse, en tanto que en registros mayores de $35^{\circ} \mathrm{C}$ y menores de $20{ }^{\circ} \mathrm{C}$ disminuye o tiende a suprimirse el comportamiento alimenticio.

Finalmente, la evidencia experimental obtenida muestra que el grado de amenaza de $B$. asper, debido a cambios térmicos asociados a la pérdida de hábitat, o al cambio climático, puede estar subvalorado en Colombia. Basándose en las evidencias aquí descritas, se sugiere ubicar a esta especie en la condición de casi amenazada (near threatened $-N T-$ ).

\section{Conclusiones}

Bothrops asper presenta respuestas ecofisiológicas diferenciales en algunos eventos de su historia de vida frente a cambios térmicos inducidos, lo cual evidencia una probable afectación de este taxón ante las variaciones climáticas actuales y la degradación progresiva de su hábitat. Ello también afecta su distribución geográfica y permitiría ubicar esta serpiente en la condición de especie casi amenazada (near threatened $-N T-$ ).

\section{Agradecimientos}

El primer autor agradece a la Facultad de Ciencias de la Universidad del Tolima por la formación recibida y al antiguo Serpentario Nacional por permitir la realización de esta investigación en sus instalaciones.

\section{Literatura Citada}

1. Alape-Girón A, Sanz L, Escolano J, Flores-Díaz M, Madrigal M, Sasa M, Calvete JJ. 2008. Snake venomics of the lancehead pitviper bothrops asper: 
geographic, individual, and ontogenetic variations. J Proteome Res 7: 3556-3571. doi: 10.1021/pr800332p

2. Angilleta MJ, Niewiarowski PH, $\mathrm{Na}$ vas $C A$. 2001. The evolution of thermal physiology in ectotherms. J Therm Biol 27: 249-268. doi: 10.1016/S03064565(01)00094-8

3. Bartholomew GA. 1979. Temperatura del cuerpo y metabolismo energético. En: Gordon MS (ed). Fisiología animal. México: Continental. p 381-470.

4. Bozinovic F. 2003. Termorregulación en reptiles: un pasado velozy un futuro lento. En: Fisiología ecológica y evolutiva. Teoría y casos de estudios en animales. Santiago de Chile: Univ. Católica de Chile. p 207-224.

5. Bravo A, Porzecanski AL. 2018. Applying critical thinking to the amphibian decline problem. Lessons in Conservation 8: 44-53.

6. Bustamante MR, Ron S, Coloma LA. 2005. Cambios en la diversidad de siete comunidades de anuros en los Andes de Ecuador. Biotropica 37: 180-189. doi: 10.1111/j.1744-7429.2005.00025.x

7. Bustos MG, Manjarrez J, CastroFranco R. 2013. Uso de microhábitat y termorregulación en Sceloporus horridus horridus (Wiegmann 1939) (Sauria: Phrynosomatidae). Acta Zool Mex 29: 153-163.

8. Campbell JA, Lamar WW. 2004. The venomous reptiles of the Western hemisphere. Ithaca, NY: Cornell University Press. 774 p.

9. Cisneros-Heredia Diego F, Touzet JM. 2004. Distribution and conservation status of Bothrops asper (Garman, 1884) in Ecuador (Squamata: Serpentes: Viperidae: Crotalinae). Herpetozoa 17: 135-141.

10. Díaz-Ricaurte JC, Cubides-Cubillos SD, Ferreto B. 2018. Bothrops asper (Garman, 1884). Catálogo de anfibios y reptiles de Colombia 4(2): 8-22.
11. Farsky SHP, Gonçalves LRC, Gutierrez JM, Correa AP, Rucavado A, Gasque P, Denise V. 2000. Bothrops asper snake venom and its metalloproteinase $\mathrm{BaP}-1$ activate the complement system. Role in leucocyte recruitment. Mediat Inflamm 9: 213-221. doi: 10.1080/09629350020025728

12. Glanville EJ, Seebacher F. 2006. Compensation for environmental change by complementary shifts of thermal sensitivity and thermoregulatory behaviour in an ectotherm. J Exp Biol 209: 4869-4877. doi: 10.1242 / jeb.02585

13. Gutiérrez JM. 2013. Snakebite envenoming in Latin America and the Caribbean. In: Clinical toxinology. Dordrecht: Springer. p. 1-20.

14. Hernández-Cuadrado EE, Murgas LDS, Badot PM, DeGiorgi F. 2014. Sinergia de cambios térmicos y de $\mathrm{pH}$ sobre la ontogenia inicial de Prochilodus lineatus. Rev Colomb Cienc Anim 6: 1-16.

15. Hernández-Cuadrado EE. 2009. Sensibilidad térmica del desempeño locomotor en anuros de un gradiente altitudinal en los andes colombianos. Tesis de Maestría. Ibagué, Colombia: Univ. del Tolima. $94 \mathrm{p}$.

16. [IUPS] International Union of Physiological Sciences. 2003. Glossary of terms for thermal biology. J Thermal Biol 28: 75-106. doi: 10.1016/S03064565(02)00055-4

17. Huang SP; Hsu Y, Tu MC. 2005. Thermal tolerance and altitudinal distribution of two Sphenomorphus lizards in Taiwan. J Therm Biol 31: 378385. doi: 10.1016/j.jtherbio.2005.11.032

18. Huey RB, Stevenson RD. 1979. Integrating thermal physiology and ecology of ectotherms: a discussion of approaches. Am Zool 19: 357-366. doi: 10.1093/icb/19.1.357

19. [INS] Instituto Nacional de Salud, Colombia. 2017. Protocolo de vigilancia en Salud Pública, accidente ofídico. Código 100.PRO-R02.40000-59V02. 
20. IUCN Red List of Threatened Species. 2018. [Internet]. Available in: http:// www.iucnredlist.org/

21. Leroi AM, Bennett AF, Lenski RE. 1994. Temperature acclimation and competitive fitness: an experimental test of the beneficial acclimation hypothesis. P Natl Acad Sci Usa 91: 1917-1921.

22. Lutterschmidt WI, Hutchison VH. 1997. The critical thermal maximum: history and critique. Can J Zool 75: 15611574. doi: 10.1139/z97-783

23. Lynch JD. 2012. El contexto de las serpientes de Colombia con un análisis de las amenazas en contra de su conservación. Rev Acad Colomb Ciencias Exactas Físicas Naturales 36: 435-449.

24. Lynch J. 2015. The role of plantations of the African palm (Elaeis guineensis) in the conservation of snakes in Colombia. Caldasia 37: 169-182. doi: http:// dx.doi.org/10.15446/caldasia.v37n1.50992

25. Mack-Wen VL, Rico LB, Alarcón JC, Pereañez $J A$. 2011. Inhibición in vitro del veneno de Bothrops asper con extractos etanólicos de Brownea ariza B. (Caesalpiniaceae). Vitae 18: 43-48.

26. Matias NR, Verrastro L. 2018. Biología térmica de Amphisbaena munoai (Squamata: Amphisbaenidae). Zoologia (Curitiba) 35: 1-9. doi: 10.3897/zoologia.35.e245740

27. Mora-Obando D, Díaz C, Angulo Y, Gutiérrez JM, Lomonte B. 2014. Role of enzymatic activity in muscle damage and cytotoxicity induced by Bothrops asper Asp49 phospholipase A2 myotoxins: are there additional effector mechanisms involved? PeerJ 2: e569. doi: 10.7717/peerj.569

28. Ortega Z, Pérez-Mellado V. 2016. Seasonal patterns of body temperature and microhabitat selection in a lacertid lizard. Acta Oecologica 77: 201-206.

29. Otero-Patiño R. 2009. Epidemiological, clinical and therapeutic aspects of Bothrops asper bites. Toxicon 54: 998-
1011. doi: https://doi.org/10.1016/ j.toxicon.2009.07.001

30. Saldarriaga-Córdoba M, Parkinson CL, Daza JM, Wuster W, Sasa M. 2017. Phylogeography of the Central American lancehead Bothrops asper (Serpentes: Viperidae). Plos One 12: e0187969. doi: 10.1371/journal.pone.0187969

31. Sasa M, Wasko K, Lamar WW. 2009. Natural history of the terciopelo Bothrops asper (Serpentes: Viperidae) in Costa Rica. Toxicon 54: 904-922. doi: 10.1016/j.toxicon.2009.06.024

32. Seebacher F, Franklin CE. 2005. Physiological mechanisms of thermoregulation in reptiles: a review. J Comp Physiol B: 175: 533-541. doi: 10.1007/ s00360-005-0007-1

33. Sherwood L, Klandorf H, Yancey PH. 2012. Animal physiology: from genes to organisms. Australia: Cengage Learning. $896 \mathrm{p}$.

34. Vélez SM, Salazar M, Acosta H, Gómez L, Rodríguez A, Correa D, Saldaña J, et al. 2017. Geographical variability of the venoms of four populations of Bothrops asper from Panama: toxicological analysis and neutralization by a polyvalent antivenom. Toxic on 132: 55-61. doi: 10.1016/ j.toxicon.2017.04.002

35. Wasko D, Sasa M. 2009. Activity patterns of a neotropical ambush predator: spatial ecology of the Fer-delance (Bothrops asper, Serpentes: Viperidae) in Costa Rica. Biotropica 41: 241-249. doi: 10.1111/j.1744-7429.2008.00464.x

36. Wilson R. 2001. Geographic variation in thermal sensitivity of jumping performance in the frog Limnodynastes peronii. J Exp Biol 204: 4227-4236.

37. Yáñez-Arenas C, Peterson AT, Rodríguez-Medina K, Barve N. 2016. Mapeando el riesgo actual y futuro de mordedura de serpiente en el nuevo mundo. Clim Chang 134: 697-711. doi: 10.1007 / s10584-015-1544-6 